\title{
Dermatófitos no Estado do Amazonas - Brasil
}

\author{
Mário Augusto Pinto de Moraes (*) \\ Instituto Evandro Chagas - Belém-Pa.
}

\begin{abstract}
SINOPSE
De 696 casos de micoses superficiais, encontrados em Manaus - Estado do Amazonas, durante um período de 7 anos (1961 - 1968), isolaram-se 515 amostras de fungos, das quais 440 pertenciam a espécie 3 co grupo dos dermatófitos. O fungo predominante foi Trichophyton tonsurans, responsável por 282 das 440 amostras, ou seja, $64 \%$ do total de dermatófitos. Contribuiu para este percentual, bastante elevado, o fato de ser $\mathbf{T}$. tonsurans quase que o único agente causador de tinha da cabeça nas crianças de $\mathrm{Ma}$ naus: em 242 casos de tinha tonsurante apareceu Gle 234 vezes, e em 30 casos de quério, 23 vezes. Nas pessoas maiores de 15 anos, todavia, o dermatófito mais encontrado foi $\mathbf{T}$. mentagrophytes; apareceu tile 106 vezes, contra apenas 16 de $\mathbf{T}$. tonsurans, em 145 amostras de dermatófitos isolados deste grupo.
\end{abstract}

\section{INTRODUÇÃO}

As dermatofitoses são de distribuição universal, porém a cada região do globo corresponde uma flora particular de dermatófitos, caracterizada pela exigência ou predominância de certas espécies. Algumas destas são cosmopolitas, variando apenas a freqüência com que aparecem nos diferentes lugares, enquanto outras têm uma distribuição geográfica bastante limitada, sendo mesmo típicas às vezes de determinadas áreas.

As espécies predominantes não somente variam no espaço, mas podem também mudar com o passar do tempo (Ajello, 1960; Degos, 1952; Kornbleet, 1960; Lostia, 1960). As forças que determinam a mudança são, segundo Ajello (1960), o clima, hábitos culturais, atividades humanas sociais e anti-sociais, migrações e o desenvolvimento da terapêutica.

Num país de grandes dimensões como o Brasil, com áreas de clima, colonização e cultura tão diferentes, já era de prever-se que a fiora dermatofítica variasse bastante de uma região para outra. Apesar dos estudos sobre o assunto não permitirem ainda a organização de um quadro completo da prevalência dos dermatófitos em todos os Estados brasileiros, já podemos hoje dizer que a flora do Norte do país é bem diversa daquela encontrada no Sul, variação devida principalmente à freqüência das espécies isoladas. Londero (1962), baseado em dados e informações obtidos em 7 Estados, mostrou que $T$. tonsurans é a espécie predominante abaixo de $15^{\circ}$ de latitude sul e $M$. canis, a espécie dominante acima de $15^{\circ}$ de latitude sul.

A presente contribuição traz os resultados das investigações micológicas feitas na cidade de Manaus - Estado do Amazonas, em casos de micoses superficiais vistos durante um período de 7 anos (1961-1968).

\section{MATERIAL E MÉTODOS}

Foram observados ao todo 696 casos, sendo 377 em menores de 15 anos e 319 em indivíduos de ou acima de 15 anos. A maioria dos pacientes compareceu a exame encaminhada pelo Ambulatório de Dermatologia da Secretaria de Saúde do Estado do Amazonas, e o restante, por várias clínicas privadas da cidade de Manaus. Houve assim representantes das diversas camadas sociais da população em geral.

O material para exame micológico — pelos e escamas - , após a colheita, era semeado em tubos com Sabouraud-glicose, ao qual se adicionara tetraciclina ou cloranfenicol, na quantidade de $50 \mathrm{mg}$ por litro do meio.

$\mathrm{Na}$ colheita do material, a experiência adquirida mostrou que, ao invés da remoção dos pelos com pinças ou das escamas diretamente com o bisturi, o melhor era umedecer primeiro o local com água esterilizada ou, o que nos pareceu mais eficaz, com álcool a $70^{\circ}$, e depois, enquanto ainda úmido, raspar toda a área afe-

(*) - Ex-pesquisador do Instituto Nacional de Pesquisas da Amazônia. 
tada com bisturi rombo. Obtinha-se dessa for ma abundante material, sem perigo das escamas se desprenderem do bisturi e ficarem perdidas. Da lâmina do bisturi o material era transferido para uma placa de vidro esterilizada, e então aproveitado para exame direto e semeadura nos tubos.

A dificuldade na obtenção de material das unhas nos levou também a desprezar os métodos aconselhados - corte com tesoura e raspagem com lima ou bisturi —, e a adotar um outro proresso que consistia em remover toda a parte diente da unha, com uma lâmina de barbear afıada, procedendo da parte não afetada em sentıdo distal, sempre procurando passar o mais próximo possível do leito ungueal. O fragmento ou fragmentos assim removidos eram depois divididos em pequenas porções para a semeadura em Sabouraud.

Com a finalidade de confirmar-se a diferença entre as amostras de $T$. mentagrophytes produtoras de pigmento, muito freqüentes, e $T$. rubrum, empregou-se o cultivo em meio Corn Meal Agar, com $1 \%$ de glicose, no qual apenas a segunda espécie é capaz de continuar produzindo o pigmento vermelho.

\section{Resultados}

Do grupo de menores de 15 anos (Tabela 1). com 377 indivíduos, isolaram-se 295 amostras de dermatófitos. Predominou nitidamente $T$. tonsurans, espécie responsável por $96,6 \%$ dos casos positivos de tinha tonsurante e $76,6 \%$ dos casos de quério. Esses casos perfaziam juntos $92 \%$ do total de positivos do grupo $T$. tonsurans foi, porém, sobrepujado por $T$. mentagrophytes nas tinhas da pele glabra não acompanhadas de tinha da cabeça, as quais constituiram, no entanto, apenas os $8 \%$ restantes do total de posiitvos. E ainda assim, a diferença não foi expressiva: $T$. mentagrophytes apareceu 11 vezes e $T$. tonsurans, 9 vezes, em um total de 23 casos.

O sexo masculino (Tabela I), entre os menores de 15 anos, teve uma participação maio: co que o sexo feminino, principalmente nas ti. nhas tonsukantes do couro cabeludo. Neste tıpo, a proporção ficou acima de 2: 1 , sendo bastante elevada também nos casos de quério e de tinha da pele glabra.
No grupo de indivíduos de ou acima de 15 anos, $T$. mentagrophytes foi o fungo mais encontradiço, excetuadas as leveduras do gênero Candida (Tabela IV). Das 4 espécies de dermatófitos isoladas - T. mentagrophytes, $T$. tensurans, $T$. rubrum e $E$. floccosum -, o primeiro apareceu, considerando-se todos os exames positivos do grupo, em $78,6 \%$ dos casos de tinha da pele glabra, em $12 \%$ dos casos de onicomicose (o gênero Candida aqui foi responsável por mais de $80 \%$ dos casos), em cerca de $70 \%$ dos casos de tinha ínguino-crural, e em cerca de $30 \%$ dos casos de tinha das extremidades (novamente o gênero Candida predominou nesta forma, sendo responsável por mais de $50 \%$ dos casos).

E. floccosum, a espécie menos freqüente (9 casos), apareceu quase que somente nas tinhas dos pés, em adultos.

Foram encontrados 4 casos de tinha da cabeça entre indivíduos com mais de 15 anos, três deles causados por $T$. tonsurans. Todos cs casos pertenciam ao sexo feminino, e em um, que revestiu a forma de um querio, a paciente contava mais de 60 anos de idade (Foto 1).

Ainda entre adultos, houve 3 casos de tinha da barba, sendo $T$. tonsurans isolado duas vezes.

\section{COMEnTÁrios}

A espécie $T$. tonsurans é a mais abundante em Manaus como agente de dermatofitoses. $\mathrm{Na}$ cidade de Belém-Estado do Pará, Moraes \& Vilela (1962) encontraram ser também $T$. tonsu-

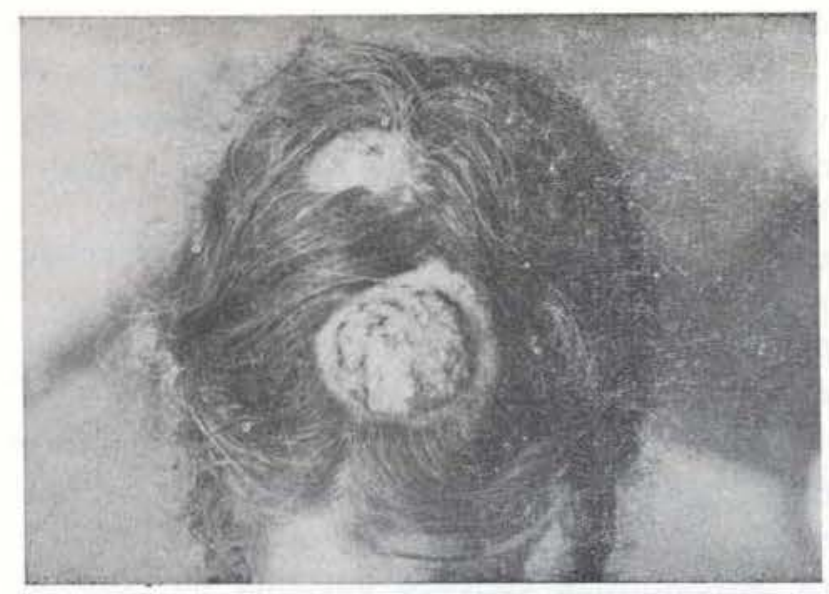

Foto 1 - Quério causado por $T$. tonsurans em mulher com mais de 60 anos de idade. 
rans o dermatófito mais freqüente: em 105 casos de tinhas da cabeça dita espécie foi isolada 104 vezes, e em 19 casos de tinhas do corpo, entre crianças, $T$. tonsurans apareceu em 15 (Tabela V). O mesmo fungo tem sido encontrado ainda como agente principal de dermatofitoses em Fortaleza, Recife e Salvador (Campos et alii, 1960/61; Campos, 1962; Cantídio, 1960; Silva: In Londero, 1962; Vieira, 1965). É a espécie predominante em todo o Norte e Nordeste do país.

Não se conhecem os fatos que levaram $T$. tensurans a instalar-se nas regiões mencionadas. Sem dúvida, foi ele importado da Europa, e a diferença na colonização talvez explique essa maior freqüência de $T$. tonsurans e das tricofitoses em geral no Norte do País, em contraste com o Sul, onde predominam as microsporias (Castro, 1928, 1929; Gonçalves et alii, 1960; Londero, 1962, 1963, 1966; Londero et alii, 1962; Magalhães, 1957; Magalhães \& Neves, 1926; Moraes \& Vilela, 1963; Pereira, 1963; Rosseti, 1941).

A preferência pelas crianças do sexo masculino é devida, certamente, à própria natureza do agente. $T$. tonsurans é um dermatófito antropofílico, transmitido entre nós quase sempre por meio de tesouras e pentes contaminados. Há, desse modo, mais chances para os meninos se contaminarem ao freqüentarem barbearias onde a limpeza e a esterilizaçäo dos instrumen tos não é prática de higiene observada.

Apesar da maior abundância de $T$. tonsurans no total e entre menores de 15 anos, não é ele. todavia, o agente mais importante das tinhas nos adultos. Predomina aqui o fungo $T$. mentagrophytes, responsável por 106 dos 145 casos de dermatofitoses encontrados nos maio-

TABELA I

TINHAS EM MENORES DE 15 ANOS - MANAUS, AMAZONAS

\begin{tabular}{|c|c|c|c|c|c|c|c|c|c|c|}
\hline TIPO & N॰ CASOS & POSIT. & - & $\%$ & MASC. & - & $\% *$ & FEM. & - & $\% *$ \\
\hline Tonsurante & 298 & 242 & & 81,2 & 133 & & 54,9 & 109 & & 45.1 \\
\hline Quério & 38 & 30 & & 78,9 & 19 & & 63,3 & 11 & & 36,7 \\
\hline Pele glabra & 41 & 23 & & 56,0 & 16 & & 69,5 & 7 & & 30.5 \\
\hline TOTAL & 377 & 295 & & 78,2 & 168 & & 56,9 & 127 & & 43,1 \\
\hline
\end{tabular}

- Percentual de casos positivos.

TABELA II

DERMATOFITOS ISOLADOS EM MENORES DE 15 ANOS - MANAUS

TIPOS DE TINHAS

\begin{tabular}{|c|c|c|c|c|c|c|c|}
\hline \multirow[t]{2}{*}{ ESPECCIE } & \multirow[t]{2}{*}{ TODOS } & \multicolumn{2}{|c|}{ TONSURANTE } & \multicolumn{2}{|c|}{ QUERIO } & \multicolumn{2}{|c|}{ PELE GLABRA } \\
\hline & & No & $\%$ & N.० & $\%$ & N. & $\%$ \\
\hline T. tonsurans & 266 & 234 & 96.6 & 23 & 76,7 & 9 & 39,3 \\
\hline$T$. mentagrophytes & 17 & 3 & 1,3 & 3 & 10,1 & 11 & 47.8 \\
\hline T. rubrum & 1 & 0 & 0,0 & 0 & 0,0 & 1 & 4,3 \\
\hline M. canis & 8 & 5 & 2,1 & 2 & 6,6 & 1 & 4,3 \\
\hline M. gypseum & 2 & 0 & 0,0 & 2 & 6,6 & 0 & 0.0 \\
\hline E. floccosum & 1 & 0 & 0,0 & 0 & 0,0 & 1 & 4,3 \\
\hline TOTAL & 295 & 242 & 100,0 & 30 & 100,0 & 23 & 100,0 \\
\hline
\end{tabular}


res de 15 anos, em Manaus. O mesmo, aliás, ¿conteceu em Belém do Pará, no inquérito já referido ( $:$ oraes \& Vilela, 1962), onde $T$. mentagrophytes foi responsável por 24 dos 29 casos estudados.

As microsporias são raras no Norte. Mi- crosporum canis foi isolado apenas 8 vezes, em Nianaus, sempre em menores de 15 anos. Microsporum gypseum, apesar de existir no solo dessa cidade (Moraes \& Vilela, 1962), apareceu apenas em dois casos, ambos sob a forma de quério.

TABELA III

TINHAS EM MENORES DE 15 ANOS - MANAUS, AMAZONAS

\begin{tabular}{|c|c|c|c|c|c|c|c|c|c|}
\hline TIPO & N० CASOS & POSIT. & - & MASC. & - & $\% *$ & FEM. & - & $\%$ \\
\hline Pele glabra & 117 & 75 & 64,1 & 50 & & 66,6 & 25 & & 33,3 \\
\hline Unhas & 56 & 41 & 73,2 & 11 & & 26,8 & 30 & & 73,1 \\
\hline Ínguino-crural & 43 & 32 & 74,4 & 26 & & 81,2 & 6 & & 18,7 \\
\hline Mãos e pés & 96 & 66 & 68.7 & 45 & & 68,1 & 21 & & 31,8 \\
\hline Outras & 7 & 6 & 85,7 & 2 & & 33,3 & 4 & & 66,6 \\
\hline TOTAL & 319 & 220 & 68,9 & 134 & & 60,9 & 86 & & 39,0 \\
\hline
\end{tabular}

- Percentual de casos positivos.

TABELA IV

DERMATÓFITOS ISOLADOS EM MENORES DE 15 ANOS - MANAUS

TIPOS DE TINHAS

\begin{tabular}{|c|c|c|c|c|c|c|c|c|c|c|c|}
\hline \multirow[t]{2}{*}{ ESPECIE } & \multirow{2}{*}{$\underset{\mathrm{N}}{\text { TODOS }}$} & \multirow{2}{*}{$\begin{array}{l}\text { PELE } \\
\mathrm{N}: 0^{\circ}\end{array}$} & \multirow{2}{*}{$\underset{\%}{\text { GLAB }}$} & \multicolumn{2}{|c|}{ UNHAS } & \multicolumn{2}{|c|}{ CRURAL } & \multicolumn{2}{|c|}{ MAOS E PÉS } & \multicolumn{2}{|c|}{ OURROS } \\
\hline & & & & N.० & $\%$ & N.० & $\%$ & N. & $\%$ & N.ㅇ & $\%$ \\
\hline T. mentag. & 106 & 59 & 78,7 & 5 & 12,2 & 22 & 68,7 & 19 & 28,7 & 1 & 16,7 \\
\hline$T$. tons. & 16 & 5 & 6,6 & 2 & 4,9 & 1 & 3,2 & 3 & 4,6 & 5 & 83,3 \\
\hline T. rubrum & 15 & 9 & 12,1 & 0 & 0,0 & 4 & 12,5 & 2 & 3,0 & 0 & 0,0 \\
\hline E. floccos. & 8 & 0 & 0,0 & 0 & 0.0 & 0 & 0,0 & 8 & 12,2 & 0 & 0,0 \\
\hline Candida sp. & 75 & 2 & 2,6 & 34 & 82.9 & 5 & 15,6 & 34 & 51,5 & 0 & 0.0 \\
\hline TOTAL & 220 & 75 & 100,0 & 41 & 100,0 & 32 & 100,0 & 66 & 100,0 & 6 & 100,0 \\
\hline
\end{tabular}

TABELA V

DERMATOFITOS EM CRIANÇAS E ADULTOS DE BELÉM DO PARA

(MORAES \& VILELA, 1962)

\begin{tabular}{|c|c|c|c|c|c|}
\hline \multirow[t]{2}{*}{ ESPECIES } & T. CAPITIS & \multicolumn{2}{|c|}{ T. CORPORIS } & CRURIS & \multirow{2}{*}{$\begin{array}{l}\text { T. PEDIS } \\
+\quad 15\end{array}$} \\
\hline & -15 & -15 & $+\quad 15$ & +15 & \\
\hline T. tonsurans & 104 & 15 & 3 & 0 & 0 \\
\hline T. mentagrophytes & 0 & 3 & 7 & 16 & 1 \\
\hline T. rubrum & 0 & 0 & 0 & 2 & 0 \\
\hline M. canis & 1 & 1 & 0 & 0 & 0 \\
\hline TOTAL & 105 & 19 & 10 & 18 & 1 \\
\hline
\end{tabular}




\section{SUMMMARY}

From 696 cases of superficial mycoses diagnosed in Manaus - State of Amazonas, within a sevenyear period (1961-1968), the author obtained 515 strains of fungi, including 440 strains which belonged to the dermatophyte group. Trichophyton tonsurans was the predominant species; in fact, it is the most common dermatophyte isolated from human cases in the States of Northern Brazil, as has been shown by other investigators. In the present work, $\mathbf{T}$. tonsurans was the etiological agent in $64 \%$ of the 440 cases of dermatophytoses. This high percentage was due mainly to the fact that $\mathbf{T}$. tonsurans is almost solely responsible for tinea among the children of Manaus. Of 242 cases of ringworm of the scalp and of 30 cases of Kerion, T. tonsurans was isolated from 234 and 23 cases respectively. In adults, however, Thichophyton mentagrophytes was the most common agent of tinea; of 145 strains of dermatophytes isolated from adults 106 were $\mathbf{T}$. mentagrophytes and 16 were $\mathbf{T}$. tonsurans.

\section{BIBLIOGRAFIA CITADA}

AJELLO, L.

1960 - Geographic distribution and prevalence of the dermatophytes. Ann. N. Y. Acad. Sci., $89: 30-38$.

CAMPos, S. T. C.

1962 - Tinhas da criança no Recife. Pulçōes Inst. Micol, Recife, 285.

CAMPos, S. T. C. ET ALII

1960/61 - Tinhas tricofíticas no Recife. Dermatol. Venezol., 2 : 165-188.

CANTídio, W.

1960 - Relatório apresentado à XVII Reunião de Dermatologistas brasileiros, Curitiba.

CASTRO, A. M.

1928 - Tinhas dos animais domésticos em São Paulo. I. Achos Inst. biol., S. Paulo, 1: 201-216.

1929 - Tinhas dos animais domésticos em São Paulo. II. Trichophycia. Archos Inst. biol., S. Paulo, 2: 163-179

Degos, R. \& Rivalier, E.

1952 - In : Précis de Mycologie. - Langeron, M. \& Vanbreuseghem, R. Paris, Masson et cie.
Gonçalves, A. P. ET aLII

1960 - Dermatófitos obscrvados no Dzpartamento de Dermatologia da Policlínica geral do Rio de Janeiro. Bras.-Med.,. Rio de Janeiro, 74 : 398-401.

KornbleEt, L. V.

1960 - The changing pattern of superficial fungous infection in New York City. Dermatologica, Basel, 120 : 185-190.

LONDERO, A. T.

1962 - The geographic distribution and prevalence of dermatophytes in Brazil. Sabouraudia, 2:108-110

1963 - Microsporum infections in Rio Grande do Sul (Brazil). Dermatol. Trop., 2: 164-167.

LONDERO, A. T. ET ALII

1962 - Os dermatófitos no interior do Rio Grande do Sul. Hospital, Rio de Janeiro, 61: 161-165.

Lostia, A. \& Rusu, C.

1960 - Studio epidemiologico sulle microsporia in Sardegna. Ross, Med. Sarda, 62: 69-73.

Magalhães, O.

1957 - Ensaios de micologia. Mem. Ins. Osw. Cruz, 55: $1-15$.

Magalhāes, O. \& Neves, A.

1926 - Ensaios de micologia. Contribuição para o estudo dos cogumelos em Belo Horizonte. Mem. Inst. Osw. Cruz, 19: 245-283.

Moraes, M. A. P. \& Vilela, E. M.

1962 - Isolamento de Microsporum gypseum e Trichophyron mentagrophytes do solo da cidade de Manaus, Amazonas. Rev. Inst. Med. Trop. S. Paulo, 4 : 299-301.

$1962 \mathrm{a}$ - Dados não publicados

PEREIRA, C. C. A.

1963 - Tinhas do couro cabeludo em São Paulo. An. bras. Dermat. 38 : 32-35.

RosSeTI, N.

1941 - Um novo problema sanitário em São Paulo. Primeiros resultados de um inquérito sobre as tinhas. Rev, Inst. Adolfo Lutz, 1: 217-303.

SILVA, M. E.

1962 - In: The geographic distribution and prevalence of dermatophytes in Brazil - Londero, A. T. Sabouraudia, 2 : 108-110.

VIEIRA, J. R.

1965 - Considerações sobre a incidência de Tinea capitis no Recife. Hospital, Rio de Janeiro, $67: 635-640$. 